\title{
MODELO DE INNOVACIÓN EDUCATIVA. UN MARCO PARA LA FORMACIÓN Y EL DESARROLLO DE UNA CULTURA DE LA INNOVACIÓN
}

\author{
(MODEL OF EDUCATIONAL INNOVATION. A FRAME FOR TRAINING \\ AND DEVELOPMENT OF A CULTURE OF THE INNOVATION)
}

\author{
Pedro Ortega Cuenca \\ María Eugenia Ramírez Solís \\ José Luis Torres Guerrero \\ Ana Emilia López Rayón \\ Citlali Yacapantli Servín Martínez \\ Liliana Suárez Téllez \\ Blanca Ruiz Hernández \\ Instituto Politécnico Nacional (México) \\ Instituto Tecnológico de Monterrey (México)
}

\section{RESUMEN}

La innovación educativa en el Instituto Politécnico Nacional es considerada como una estrategia para avanzar en el logro de los fines institucionales. Su trayectoria aún es joven y requiere ampliarse, consolidarse y penetrar en todos los ámbitos de la institución, logrando así desarrollar una nueva cultura. El modelo de innovación educativa del IPN proporciona un marco que permite contribuir a la realización de auténticos proyectos de innovación. En este trabajo se presentan los criterios y fases que caracterizan a la innovación educativa y guían el proceso mismo de la innovación, así como las figuras que en ella participan. También se abordan aquellos elementos que permitirán formar y desarrollar una cultura de la innovación que distinga a la institución por su carácter innovador.

Palabras clave: innovación educativa, modelo de innovación educativa, cambio educativo, cultura de la innovación, reforma académica, investigación educativa. 


\begin{abstract}
Educational Innovation at the IPN is considered a strategy in the advancement to achieve the objectives of the institution. Although, it is a relatively new concept, which needs to become established and to be consolidated, it is expected to be extended to all areas of the Institute eventually, for the creation of a new culture of innovation. The IPN model of innovation provides us with a frame which allows authentic innovative projects. This work presents the criteria and outlines the phases that characterize these projects of innovation, as well as providing some elements to create and develop the culture of innovation that would characterize the IPN
\end{abstract}

Key words: educational innovation, educational innovation model, educational change, culture of innovation, academic reform, educational research.

El Instituto Politécnico Nacional (IPN) de México cumplió 70 años en 2006. El IPN ofrece 203 programas educativos de los niveles medio superior, superior y postgrado en los que estudian 179 mil estudiantes y cuenta con cerca de 24 mil profesores y personal de apoyo.

En 2001, el Instituto emprendió una Reforma Académica que reconoce como su misión: "El IPN es la institución educativa laica, gratuita de Estado, rectora de la educación tecnológica pública en México, líder en la generación, aplicación, difusión y transferencia del conocimiento científico y tecnológico, creada para contribuir al desarrollo económico, social y político de la nación. Para lograrlo, su comunidad forma integralmente profesionales en los niveles medio superior, superior y postgrado, realiza investigación y extiende a la sociedad sus resultados, con calidad, responsabilidad, ética, tolerancia y compromiso social”.

El IPN, para avanzar en la realización de su visión al 2025 y cumplir con "el papel de rectoría de la educación tecnológica pública que la sociedad le ha conferido" y que "debe ser asumido ahora desde un punto de vista más cualitativo que cuantitativo" (IPN, 2004 ${ }^{\mathrm{a}}$, pp 43-44), ha definido seis líneas estratégicas que orientan las acciones de cada unidad académica: atención a la demanda; innovación y calidad en la formación; responsabilidad y relación con el entorno; conocimiento para el desarrollo del país; atención a la comunidad, y una nueva gestión institucional. Para realizar las acciones que permitan atender a cada una de estas líneas estratégicas hay que destacar que la calidad y la innovación son los principios que guían a la institución. Los esfuerzos de innovación se dirigirán a la mejora de los aspectos que a continuación se presentan:

- Planes de estudio centrados en el aprendizaje, que garanticen flexibilidad para contar con salidas laterales, señalen trayectorias académicas, reconozcan 
las diferencias individuales de los estudiantes y favorezcan la movilidad institucional entre otras instituciones nacionales e internacionales.

- Una oferta educativa congruente con los principios del Modelo Educativo, pertinente a las necesidades sociales actuales y con visión prospectiva para arribar al futuro mediato e inmediato.

- Alumnos en procesos de atención individualizada, formados en ambientes que permitan deconstruir y construir conocimientos y alternativas de solución a problemas del entorno, con participación responsable en su proceso de formación integral y capaces de diseñar su propio plan de vida.

- Reconceptualización de la docencia, orientando las prácticas de los profesores como mediadores del aprendizaje; docentes actualizados tanto en lo pedagógico como en el campo de conocimientos de su disciplina, vinculados con el sector productivo e integrado en redes académicas nacionales e internacionales.

- Unidades académicas conceptualizadas como comunidades de aprendizaje, con nuevas estructuras que permitan la colaboración horizontal entre sus miembros; con programas estratégicos que integren las funciones sustantivas, con relaciones sólidas en el entorno social y laboral.

- Prácticas de evaluación internas y externas que aseguren la calidad de sus egresados y de los servicios ofrecidos por la institución.

- Consolidación del campus virtual politécnico como una estrategia para transmitir conocimientos a la población y mejorar la cobertura, la pertinencia y la equidad.

- Programas de investigación que integren la generación, la transmisión y la aplicación del conocimiento.

Se han publicado 19 Materiales para la Reforma Académica (IPN, 2004). Entre ellos destacan el Modelo Educativo (IPN, 2004a) y el Modelo de Integración Social (IPN, 2004b), que constituyen la principal referencia del quehacer del Instituto. Ambos modelos buscan identificar los caminos para avanzar hacia mayores niveles de calidad y pertinencia; son el eje conceptual de la transformación institucional y, al mismo tiempo, constituyen las guías para conducir el trabajo cotidiano de la comunidad politécnica.

El Modelo Educativo propone una concepción del proceso educativo que contrasta fuertemente con las prácticas actuales del Instituto. En este modelo se promueve una formación integral y de alta calidad, centrada en el aprendizaje del estudiante. Para lograr esto se requiere de programas formativos flexibles que incorporen la posibilidad de tránsito entre modalidades, programas, niveles y 
unidades académicas, así como la diversificación y el robustecimiento de los espacios de aprendizaje, además de cambios sustanciales en los enfoques didácticos que propicien una cultura de la innovación, la capacidad creativa e impregnen todos los procesos con el uso responsable de las TIC. La formación que resulte de la aplicación del modelo debe facultar a sus egresados para seguir aprendiendo a lo largo de toda la vida y para sobresalir en el ejercicio de su profesión en el país y fuera de él. El modelo no se limita a la formación, sino que se ocupa de las otras funciones, como la investigación, la vinculación, la extensión y la difusión, intensificando la relación del Instituto con la sociedad a la que sirve.

Por su parte, el Modelo de Integración Social define la misión social del Instituto y su relación con los distintos sectores de la sociedad como una interacción responsable y enriquecedora, que propicia la participación conjunta en la identificación de problemas y en los planes y acciones para darles solución. El Modelo de Integración Social retoma, redefine y precisa las funciones tradicionales de vinculación y extensión mediante acciones acordes con las demandas de la sociedad actual, como la cooperación interinstitucional y la internacionalización, propiciando innovaciones en las formas de organización del trabajo al interior del IPN, y la formación y la consolidación de cuerpos colegiados que estén en condiciones de establecer una vinculación con el entorno, de manera creativa, pertinente y mutuamente provechosa.

En 2005, como parte de los procesos de Reforma, el IPN realiza una reestructuración administrativa, que responde a la operación del Modelo Educativo propuesto; en este contexto comienza a operar el Centro de Formación e Innovación Educativa (CFIE) del IPN. En el Acuerdo de su creación se establecen dos objetivos: I. Coordinar, concertar, fomentar, impulsar, evaluar la participación de los órganos responsables de la formación y la innovación educativa, que facilite la coordinación de acciones para incidir en la mejora del desempeño del personal del Instituto y fortalecer la calidad y pertinencia de las funciones institucionales, y II. Establecer programas para la formación de personal de acuerdo con las necesidades de el Nuevo Modelo Educativo y el Modelo de Integración Social. En la misión del CFIE se afirma que "es la instancia dependiente de la Secretaría Académica del Instituto Politécnico Nacional, que dirige el desarrollo del talento humano desde, en, a través y para la innovación educativa, que consolide, con compromiso social, el cambio sistemático y participativo en el quehacer Institucional” (CFIE, 2006).

Los antecedentes de innovaciones educativas en el IPN comienzan prácticamente con la Reforma Académica. En el marco institucional, por lo que toca a sus 
reglamentos, no hay referencias explícitas, aparte de las que corresponden al CFIE, ya mencionadas anteriormente. Por lo que se refiere al Modelo Educativo del IPN, el papel de la innovación se destaca con tres énfasis como un aspecto de la sociedad del conocimiento, como una de las capacidades que se debe considerar en la formación de los estudiantes y como una estrategia institucional para el logro de sus objetivos. En los diagnósticos comparativos (IPN, 2004c, 2004d, 2004e), que se realizaron para determinar el posicionamiento estratégico del IPN en el sistema educativo nacional, no se utilizó la innovación educativa como categoría aunque algunos indicadores se pueden considerar relacionados con ella.

Por otro lado, la Secretaría de Educación Pública (SEP), como resultado de cinco convocatorias, ha acumulado un conjunto de prácticas innovadoras reconocidas para su consulta en Internet (SEP, 2005), clasificadas en seis categorías: Académicas, Administrativas, Científicas, Informáticas, de Investigación y Tecnológicas. Las prácticas reconocidas por la SEP se inscriben en el Programa Estratégico de Innovación y Calidad y tienen como referencia el modelo correspondiente. Entre estas prácticas reconocidas hay más de 30 que corresponden a miembros de la comunidad politécnica. Sin embargo, la mayoría de dichas prácticas han sido promovidas por directivos, lo que suele constituir un buen principio pero no garantiza un cambio auténtico en las innovaciones académicas, mientras los docentes no se hayan convencido de los beneficios del cambio.

Con estos antecedentes, tomando en consideración que las innovaciones educativas tienen ya una historia de décadas en otros países, se diseñó un Modelo de Innovación Educativa para el IPN, que permitiera sentar las bases organizacionales adecuadas para desarrollar los muchos proyectos de innovación que requiere la Reforma Académica para lograr sus ambiciosos, pero pertinentes, objetivos.

En los estudios sobre las innovaciones educativas se ha insistido bastante en el carácter culturalmente determinado de las innovaciones y de los riesgos de las transferencias ingenuas de modelos y prácticas exitosas de una región a otra. A pesar del atractivo de modelos de innovación innovadores en sí mismos, como los basados en metáforas, considerando la organización y las características de la comunidad politécnica, se ha preferido diseñar un modelo más convencional, que pueda servir de orientación para la realización exitosa, en los tiempos que requieran, de innovaciones que surjan de cualquier iniciativa que se pueda encauzar para llevar agua al molino de la Reforma Académica y que sirva de punto de partida para la formación de una cultura de la innovación. 


\section{LA NOCIÓN DE INNOVACIÓN EDUCATIVA}

Una forma ilustrativa de ver la innovación es a través del juego convencional de preguntas: ¿̇qué?, ¿̇por qué?, ¿̇para qué?, ¿̇dónde?, etc. Ahora se sabe que no todas las respuestas arrojan información igualmente importante desde el punto de vista de la innovación, aunque, claro, todas importan porque, si bien se responden por separado, sólo adquieren cabal sentido cuando se integran en un todo.

La naturaleza de la innovación es, sin duda, un aspecto fundamental del proceso y para caracterizarla se han usado varios marcos. Un cambio con mejora, con respecto a un objetivo previamente determinado, es el elemento común, aunque el cambio se puede referir a una idea, un material, una práctica, un contenido, alguna metodología, un patrón cultural, una relación entre las personas o instancias que participan en el hecho educativo, la forma de aplicar una norma, un procedimiento administrativo, un artefacto organizacional o una creencia o valor, sin ánimo de ser exhaustivos, pues hay una gran diversidad de experiencias que pueden ostentar el título de innovación educativa.

El cambio con mejora de cualquiera de estos aspectos educativos implica una situación original y otra, distinta, que será el resultado de la innovación, cuando, en el plano personal, se ha interiorizado y, en el organizacional, se ha institucionalizado, es decir, se ha convertido en la nueva normalidad, lo nuevo se vuelve común, ordinario.

El porqué aporta parte del motivo de la innovación, la justificación del promotor, que puede ser un profesor o un directivo, o algún grupo en el que intervengan unos u otros. La razón que los mueve puede surgir de la insatisfacción con respecto a algún aspecto que sea responsabilidad suya, o en el que tengan influencia, pero también puede ser una exigencia institucional, como suele ocurrir en las reformas educativas. También aquí, como en otros ámbitos, la otra parte del motivo, lo que le da sentido a una innovación, es el fin con el que se realiza, el para qué.

La innovación ocurre en algún espacio educativo, un centro, una institución, un departamento administrativo, un aula, un cuerpo académico. En un sistema, cualquiera de los espacios tiene relación con varios elementos o subsistemas, por lo que el contexto donde ocurre la innovación, con su cultura, su clima, sus resistencias, es uno de los factores que se han revelado como más influyentes en el éxito de una innovación educativa. El contexto es tan determinante que lo que en uno puede ser innovador, en otro, no lo es, estableciendo el carácter mismo de innovación de una experiencia. 
Así mismo, el contexto influye fuertemente en la viabilidad de una innovación. En particular, la cultura organizacional es un aspecto fundamental para el desarrollo de una innovación. No es fácil modificar los hábitos que se han formado a partir de la experiencia, aún cuando la práctica cotidiana demuestre sistemáticamente su ineficacia. En las reglas no escritas está el principal reto de la innovación y, para saber si es posible hacerla, es necesario estudiar la evolución de la cultura organizacional y diseñar estrategias que la conduzcan a formar una cultura de la innovación.

La unidad básica de cambio que se ha revelado como la más adecuada para la realización de innovaciones con impacto en los procesos de formación es el centro escolar o la unidad académica (Tejada, 1998). En ese nivel es posible hacer planes, diseñar estrategias articuladas y evaluar los resultados con respecto a objetivos bien definidos, con un grado importante de autonomía y diversas instancias a las que hay que rendir cuentas. La unidad académica tiene una cultura reconocible y comprende subsistemas, como el aula o la academia, con problemas identificables que se pueden resolver mediante proyectos de innovación. En la unidad académica se pueden atender las demandas sociales e integrar en los proyectos a otros actores, como padres de familia, representantes de instancias gubernamentales, empresarios y organizaciones sociales, para robustecer la vinculación de la escuela con su entorno.

Las innovaciones parten de una crítica a la situación original. Una crítica que, cuando se plantea la necesidad del cambio, suele dejar personas agraviadas. Personas que han contribuido, por acción o por omisión, a crear un problema y que, si se quiere resolver el problema, habrá que ganar para la innovación. Quien promueve una innovación no puede ser ingenuo a este respecto, debe servirse de los conocimientos sobre procesos de innovación similares reportados y, sobre todo, del conocimiento del sistema en donde se quiere realizar la innovación. Quienes subestiman la magnitud del problema o reducen la innovación a un número insuficiente de dimensiones corren el riesgo de consolidar la resistencia a la innovación, volviéndola antipática e inviable. Cuando las innovaciones se promueven desde la institución, la presión y el apoyo han sido factores influyentes en su éxito. Fullan y Stiegelbauer (1997) han advertido que la presión sin apoyo lleva a la resistencia y la enajenación en tanto que el apoyo que no va acompañado de presión conduce a divagaciones y al desperdicio de recursos.

Uno de los factores más importantes para el éxito de una innovación educativa es la forma en que los diversos actores que intervienen en el proceso interpretan y redefinen los cambios que conlleva la innovación. Los actores creen, sienten, razonan, 
hacen e interactúan entre sí y con la innovación. Las personas y las organizaciones aprenden en el proceso de innovación.

La aceptación de las innovaciones, desde el punto de vista de las personas que participan depende de varios factores, como el hecho de que reconozcan en la innovación la solución a un problema que les interesa resolver. Otro factor es el reconocimiento del carácter profesional de su quehacer, en el sentido de que hay argumentos sólidos, basados en un saber compartido, que sustentan la toma de decisiones.

Los cambios auténticos suelen ser lentos, los períodos que deben transcurrir para que las personas interioricen los cambios y las innovaciones se institucionalicen se miden en lustros o décadas. Para disponer de evidencias auténticas de los resultados de los procesos formativos, de la generación y gestión del conocimiento, deben transcurrir tiempos prolongados. La decisión de innovar no se puede tomar a la ligera porque las consecuencias del éxito o el fracaso de la innovación se vivirán durante varios años.

El proceso de innovación requiere de una evaluación continua para que haya evidencias de que los cambios apuntan al logro de los objetivos planteados. El enfoque de sistemas contribuye a que las evaluaciones aporten datos de diferentes niveles, particularmente sobre relaciones y relaciones de relaciones, sin olvidar que son el contexto y la insoslayable dimensión humana los que dan significado a esta información en el sistema educativo.

\section{EL MODELO DE INNOVACIÓN EDUCATIVA DEL CFIE}

El Modelo de Innovación Educativa proporciona un marco para realizar auténticas innovaciones y para la formación y el desarrollo de una cultura de la innovación. Precisa las condiciones necesarias para que las innovaciones puedan llegar hasta la institucionalización e interiorización.

El Modelo de Innovación Educativa del CFIE tiene dos partes. La primera parte comienza con la decisión de innovar, que es un proceso en sí mismo e incluye doce criterios para caracterizar una innovación educativa. Después de la decisión de innovar hay ocho fases que guían el proceso de innovación. En la segunda parte del modelo se proponen una serie de criterios que tienen su origen en la investigación reportada sobre innovaciones educativas. Esta segunda parte se aplicará cuando se 
decida investigar el proceso de innovación. La investigación se puede hacer sobre cualquier innovación pero, como toda investigación, se debe ubicar en un campo reconocido. La investigación tiene como propósito generar un conocimiento sobre las innovaciones y la cultura de la innovación en el IPN, un conocimiento que no por local sea menos riguroso, sólo así será útil para el IPN y comunicable al resto de la comunidad que se ocupa del campo de la innovación educativa.

\section{LOS SUPUESTOS PARA LA INNOVACIÓN EDUCATIVA}

Los supuestos para la Innovación Educativa del IPN ayudan a fijar la dirección en la que se habrán de orientar los esfuerzos. Sólo a partir de estos supuestos adquieren sentido los marcos del modelo y los proyectos de innovación que lo tomen como referencia.

- Los problemas importantes de la educación son problemas de sistema por lo que requieren, para avanzar en su solución, un enfoque sistémico. Es decir, se trata de un conjunto complejo de elementos, relaciones, mecanismos e indicadores que interactúan para lograr un cambio que conduzca a construir un sistema que evolucione ordenadamente y sea eficiente. Estas características del modelo deben aparecer explícitamente en los proyectos de innovación particulares.

- Para avanzar en la solución de problemas tan complejos como los que enfrenta un sistema educativo se necesita reconocer que no es el individuo sino el cuerpo académico (docente, directivo, de investigación, de personal de apoyo), organizado según el modelo profesional, el protagonista de las transformaciones del sistema. La docencia actualmente es una práctica tan compleja que un individuo sólo puede ejercerla con alguna probabilidad de éxito si cuenta con el respaldo de una comunidad profesional bien organizada. Lo mismo ocurre con las otras figuras, directivo, personal de apoyo e investigador.

- Una de las tendencias actuales, derivadas de la incorporación de la tecnología y de la investigación sobre los ambientes de aprendizaje, señala que las estrategias para el mejoramiento de la educación se deben ocupar preferentemente del aprendizaje, de lo que logra el estudiante más que de lo que hace el profesor. Se trata de mejorar los espacios de aprendizaje escolarizados destacando la participación del profesor y, además, brindar a los estudiantes la oportunidad de que se responsabilicen de su aprendizaje y logren cierto nivel de autonomía en sus necesidades de aprendizaje. Esto es, garantizar las condiciones y crear ambientes propicios en los que 
el estudiante tenga el control de partes del proceso. Hay un énfasis en el mejoramiento de la calidad del aprendizaje, según indicadores diversos, en sus estudiantes. Esta mejora debe ser documentable tanto según los mecanismos de evaluación internos, como según los estándares externos pertinentes.

- Las decisiones en el sistema se deben tomar considerando explícitamente el modelo subyacente y los datos que se utilizan. Es indispensable un sistema de evaluación válido, confiable y transparente con el uso de los resultados de la investigación pertinentes. Una de las características del quehacer profesional es que se usa un conocimiento específico del campo para la toma de decisiones en el ejercicio de la profesión.

- La viabilidad de los planes de innovación que contribuyan a concretar la visión que se formula en las prospectivas del IPN depende de un factor fundamental: la conformación de redes de personal de apoyo, docentes, directivos e investigadores capaces de responsabilizarse de los proyectos de innovación que requiere la Reforma y de articular sus metas con las instancias correspondientes para avanzar en el logro de los objetivos de los grupos y de la institución. Se trata de consolidar la autonomía de los individuos, y de las diversas redes de propósito específico de las que forma parte, al mismo tiempo que se incorporan mecanismos de rendición de cuentas que garanticen la efectividad de los métodos que emplean en el cumplimiento de sus funciones.

\section{LOS CRITERIOS Y LAS FASES DEL MODELO DE INNOVACIÓN EDUCATIVA}

El primer supuesto para la innovación educativa destaca su carácter sistémico, como Havelock y Huberman (1977) señalaron en su obra sobre la innovación educativa en los países en desarrollo, la teoría de sistemas es un marco adecuado para los proyectos de innovación educativa. Además, consideran las innovaciones como procesos de solución de problemas en un sistema que se encuentra dentro de otro sistema, que a su vez es parte de un sistema mayor. Según estos autores, el éxito de una innovación depende de la capacidad para articular la dependencia recíproca entre los tres niveles del sistema: el sistema social, el sistema educativo y el proyecto educativo innovador. Considerar la innovación como sistema permite establecer relaciones entre los elementos de los subsistemas y entre estos mismos. Los equilibrios y desequilibrios en distintos niveles pueden señalar el origen y la solución de algunos problemas que surgen en la vida de las innovaciones. 
Como se afirma en la presentación de la Red Innovemos de la UNESCO (2001), las innovaciones educativas dependen de diversos factores, fundamentalmente el contexto, los patrones culturales específicos, el campo de conocimiento y la visión de la educación en la que se enmarcan. Señalan que "en la actualidad, la mayoría de los enfoques sobre innovación educativa coinciden en que no existe un único modelo innovador, sino múltiples innovaciones culturalmente determinadas". Así, para delimitar el campo de la innovación educativa, en lugar de una definición de innovación educativa se proponen una serie de criterios que permitan caracterizarla aportando la información indispensable para garantizar que se trata realmente de una innovación y que tendrá razonables probabilidades de éxito. Los criterios son "objeto de revisión constante y se aplican con flexibilidad".

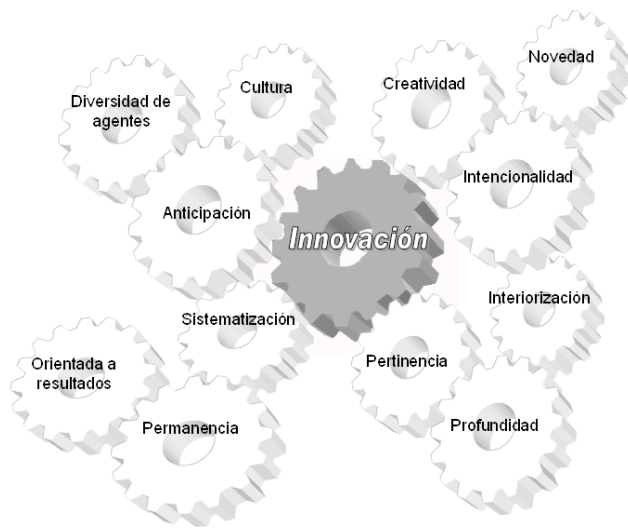

Figura 1. La noción de innovación educativa.

En el Modelo de Innovación Educativa del IPN se formulan doce criterios para caracterizar una innovación educativa, que se ilustran en la figura anterior y se presentan en la tabla siguiente. Estos criterios están basados en los de la Red Innovemos de la UNESCO y en los que proponen Blanco y Messina (2000) en su Estado del arte en América Latina, publicado por la UNESCO y el Convenio Andrés Bello. 


\begin{tabular}{|c|c|}
\hline Criterio & Descripción \\
\hline 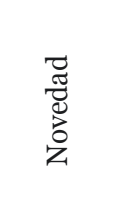 & $\begin{array}{l}\text { Una innovación introduce algo nuevo en una situación dada, algo nuevo que propicia una } \\
\text { mejora con respecto a una situación bien definida. No es necesario que sea una invención } \\
\text { pero conlleva una manera diferente de configurar y vincular los elementos que son objeto } \\
\text { de la innovación. La innovación es una creación relativa a un contexto, a una situación } \\
\text { con una tradición y unos hábitos. La innovación generalmente tiene precedentes que se } \\
\text { aprovechan como palanca para transformar la situación dada. }\end{array}$ \\
\hline 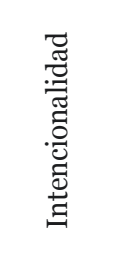 & $\begin{array}{l}\text { La innovación tiene un carácter intencional, es un cambio que deliberadamente se propone } \\
\text { lograr una mejora. Una intención congruente con el marco institucional. Los cambios } \\
\text { que ocurren sin una intención y una planeación explícitas no se pueden considerar } \\
\text { como innovaciones. Innovar es un proceso que puede tomar cursos muy diferentes } \\
\text { que difícilmente pueden predecirse de antemano. En este sentido, la planeación ha de } \\
\text { considerarse también como un proceso constante, e irse modificando en función de la } \\
\text { dinámica que acontece en la práctica. }\end{array}$ \\
\hline 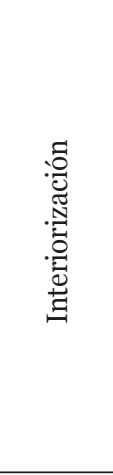 & $\begin{array}{l}\text { La innovación implica una aceptación y apropiación del cambio por parte de las personas } \\
\text { que han de llevarlo a cabo. Como punto de partida, es necesario un acuerdo entre los } \\
\text { responsables de la innovación en cuanto a los objetivos que se persiguen. } \\
\text { La mejora que representa la innovación ha de responder a los intereses de todos los } \\
\text { involucrados para que su impacto sea significativo y los cambios que produzca tengan } \\
\text { cierta permanencia. La apropiación será mayor en la medida en que la participación se } \\
\text { reconozca como valiosa. } \\
\text { Las personas, individualmente o en grupo, no pueden ser ejecutores neutros. Hay una } \\
\text { interacción entre personas, ideas y otros factores, que redefine la innovación en función } \\
\text { de las percepciones inmediatas con respecto a un marco de conocimientos y creencias. } \\
\text { En términos individuales, los cambios corresponden a un desarrollo de la personalidad, } \\
\text { en el ser, el saber y el hacer, en un proceso de interiorización de la innovación. Para la } \\
\text { institución, se traduce en cambios en la cultura organizacional. }\end{array}$ \\
\hline 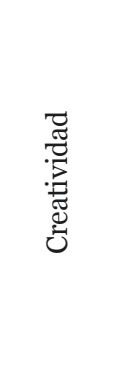 & $\begin{array}{l}\text { Considera explícitamente el surgimiento de iniciativas y la disposición para identificar } \\
\text { y definir problemas resolubles en todo el proceso. Nisbet (De la Torre, 1997) define la } \\
\text { creatividad de una escuela como la capacidad para adoptar, adaptar, generar o rechazar } \\
\text { las innovaciones. La creatividad se refleja en la capacidad para identificar mejoras, fijar } \\
\text { metas y diseñar estrategias que aprovechen los recursos disponibles para lograrlas. } \\
\text { El carácter complejo de las innovaciones educativas hace que, en ocasiones, en la } \\
\text { realización de un proyecto de innovación surjan, en la práctica, innovaciones distintas } \\
\text { dependiendo de las particularidades del contexto, que constituye un escenario único. Así, } \\
\text { debido a su complejidad, las innovaciones requieren de innovaciones dentro de la misma } \\
\text { innovación. }\end{array}$ \\
\hline 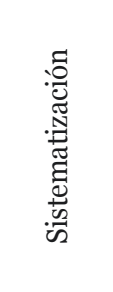 & $\begin{array}{l}\text { La innovación es una acción planeada y sistemática que involucra procesos de evaluación } \\
\text { y reflexión crítica acerca de la práctica y la innovación misma. La sistematización del } \\
\text { proceso de innovación permite generar información contextualizada que sirve de base } \\
\text { para la toma de decisiones fundamentadas. A pesar de que no es posible replicar las } \\
\text { innovaciones sin modificarlas porque cada escenario es irrepetible, hay que aprovechar, } \\
\text { sin desnaturalizar las experiencias, los aprendizajes que dejan. Una sistematización bien } \\
\text { articulada con respecto a marcos explícitos brinda la oportunidad de generar teoría acerca } \\
\text { de la práctica y mantener actualizada la información sobre las innovaciones. }\end{array}$ \\
\hline
\end{tabular}




\begin{tabular}{|c|c|}
\hline Criterio & Descripción \\
\hline 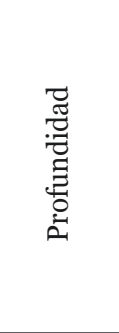 & $\begin{array}{l}\text { La innovación genera cambios en las concepciones, las actitudes y las prácticas } \\
\text { educativas. } \\
\text { La innovación implica una auténtica transformación. Una ruptura de los equilibrios } \\
\text { aceptados en las estructuras, que constituyen el funcionamiento rutinario, la situación } \\
\text { que se quiere cambiar. Cuando no se altera este funcionamiento rutinario, sólo se puede } \\
\text { hablar de ajustes, no de innovación. } \\
\text { La innovación no sólo produce cambios profundos en las instituciones, sus procedimientos } \\
\text { y sus materiales, también transforma a los actores educativos, sean éstos personas o } \\
\text { sujetos colectivos. }\end{array}$ \\
\hline 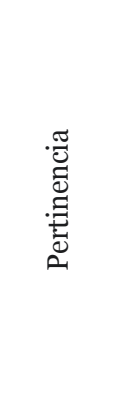 & $\begin{array}{l}\text { La innovación es pertinente al contexto socioeducativo. El contexto se reconoce como uno } \\
\text { de los factores más importantes en el éxito de las innovaciones, por lo que se deben tomar } \\
\text { en cuenta sus características, haciendo de la innovación una solución a una problemática } \\
\text { bien definida. } \\
\text { El contexto es tan determinante que lo que en uno puede ser innovador, en otro, no lo } \\
\text { es, determinando el carácter mismo de innovación de una experiencia. La tentación de } \\
\text { importar innovaciones de manera simplista es un riesgo si los contextos no son similares } \\
\text { en aquellos aspectos que tienen relación con el proceso de innovación. La innovación } \\
\text { misma puede cambiar significativamente cuando cambia el contexto, obligando a una } \\
\text { redefinición de aspectos sustantivos de la innovación pues los problemas no suelen ser los } \\
\text { mismos ni se resuelven de la misma manera en contextos distintos. }\end{array}$ \\
\hline 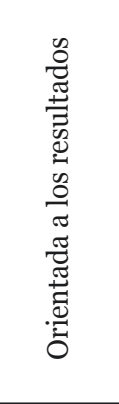 & $\begin{array}{l}\text { La innovación no es un fin en sí misma, sino un medio para lograr mejor los fines de } \\
\text { la educación. Los resultados de un sistema educativo son diversos pero se pueden } \\
\text { destacar la formación, la generación de conocimiento y la vinculación con la sociedad. Las } \\
\text { innovaciones entonces producirán una mejora en los aprendizajes de los alumnos, en la } \\
\text { gestión del conocimiento y en los proyectos conjuntos y en los convenios concretos que se } \\
\text { establecen entre las escuelas y las industrias, el gobierno y las organizaciones sociales. } \\
\text { La innovación ha de servir para mejorar la calidad y la equidad del sistema educativo. } \\
\text { En cuanto a la calidad, se trata de evaluar, con un sistema de indicadores auténticos, } \\
\text { válidos y confiables, los resultados de la innovación. La equidad no se limita a la igualdad } \\
\text { de oportunidades, incluye también la atención que se debe prestar a la diversidad en el } \\
\text { cumplimiento de las funciones sustantivas de los sistemas educativos. }\end{array}$ \\
\hline 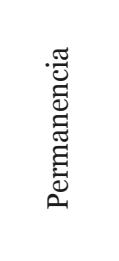 & $\begin{array}{l}\text { Se ha mantenido durante el tiempo necesario para convertirse en la nueva normalidad. } \\
\text { La complejidad de la innovación implica cambios en distintos niveles que requieren de } \\
\text { tiempos diversos para que ocurran y se consoliden. Para que estos cambios se constituyan } \\
\text { en la nueva normalidad, interiorizándose en las personas e institucionalizándose en el } \\
\text { sistema, hay una relación no lineal entre la consolidación de los cambios y el tiempo } \\
\text { transcurrido. Sin embargo, la institucionalización de una innovación no constituye una } \\
\text { garantía de su permanencia porque los procesos no son irreversibles. }\end{array}$ \\
\hline 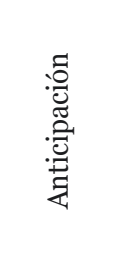 & $\begin{array}{l}\text { En una innovación educativa se vislumbra claramente cuáles son las características de } \\
\text { la situación que se quiere lograr. Sin embargo, hay que considerar que las innovaciones } \\
\text { educativas, como resultado de un proceso interactivo en el que pueden surgir imprevistos, } \\
\text { suelen seguir caminos diferentes en la práctica. Cuando los objetivos son claros, se pueden } \\
\text { definir problemas nuevos en el trayecto y resolverlos para seguir, de manera flexible, } \\
\text { tratando de lograr el cambio deseado pero con la disposición de modificar los términos de } \\
\text { la innovación cuando los cambios en el contexto así lo exigen. }\end{array}$ \\
\hline
\end{tabular}




\begin{tabular}{|c|c|}
\hline Criterio & Descripción \\
\hline $\begin{array}{l}\frac{\pi}{\Xi} \\
\frac{E}{\Xi}\end{array}$ & $\begin{array}{l}\text { La innovación produce cambios tanto en las concepciones como en la práctica. Estos } \\
\text { cambios contribuyen a formar una actitud abierta a nuevos cambios, una actitud que } \\
\text { no permita que la misma innovación se vuelva rígida. Las reformas no siempre logran } \\
\text { transformar realmente la cultura de las escuelas. El discurso del cambio que aporta la } \\
\text { reforma requiere del complemento de las acciones efectivas que aportan las innovaciones. } \\
\text { Los cambios y la incertidumbre que conllevan son parte de la normalidad en un sistema } \\
\text { complejo. Así como la reflexión sistemática sobre qué cambios realizar para mejorar el } \\
\text { funcionamiento del sistema y cómo hacerlo aprovechando la sinergia de los subsistemas. } \\
\text { Otras características importantes de esta cultura son la colaboración, como individuos } \\
\text { y como parte de distintas redes, y la capacidad de administrar los conflictos que } \\
\text { inevitablemente surgen en los procesos de innovación. Así mismo, la capacidad para } \\
\text { diagnosticar su situación, diseñar y poner en movimiento planes de acción, y evaluar tanto } \\
\text { su instrumentación como sus resultados. Por el carácter único de las innovaciones, no se } \\
\text { puede perder de vista la importancia de fortalecer la capacidad de innovar. Cuando no } \\
\text { se considera explícitamente el difícil objetivo de formar y desarrollar una cultura de la } \\
\text { innovación se corre el riesgo de que cuando desaparezcan los innovadores se acaben las } \\
\text { innovaciones. }\end{array}$ \\
\hline 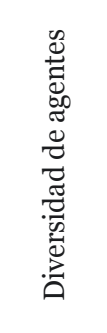 & $\begin{array}{l}\text { La diversidad de los agentes que participan en la red responsable de la innovación permite } \\
\text { la articulación de los esfuerzos en las diversas dimensiones que atañen a la innovación. La } \\
\text { colaboración de agentes diversos en la red responsable es compleja y tiene que resolver } \\
\text { los conflictos que surgen por el contraste de perspectivas distintas, pero también potencia } \\
\text { la capacidad de la red para enfrentar problemas multidimensionales que no se pueden } \\
\text { reducir a las perspectivas particulares de los individuos. Las innovaciones suelen requerir el } \\
\text { acceso a bases de datos y fuentes especializadas que los investigadores y directivos pueden } \\
\text { conseguir más fácilmente. Además, la inclusión de directivos en las redes responsables de } \\
\text { las innovaciones contribuye a fortalecer liderazgos académicos en las figuras directivas. }\end{array}$ \\
\hline
\end{tabular}

Tabla 1. Los doce criterios para caracterizar una innovación educativa del Modelo de Innovación Educativa del IPN. Basados en los criterios de la Red Innovemos de la UNESCO (2001) y en Blanco y Messina (200o).

En menos de medio siglo, periodo en el que se ha venido estudiando la innovación como fenómeno educativo, se ha generado una enorme cantidad de conocimiento sobre las muchas dimensiones de este fenómeno. La mayoría de los estudiosos concuerdan en considerar la innovación como culturalmente determinada y, por lo tanto, difícil de enmarcar en un modelo lineal debido a la circularidad y simultaneidad de algunas de sus fases, y a la necesidad de colaboración de agentes diversos. Esto ha motivado que la innovación educativa se caracterice por medio de criterios.

El Modelo de Innovación Educativa consta de dos partes. La primera, en fases, se usa en cualquier innovación, pero puede servir particularmente en aquellas innovaciones que surgen de la iniciativa de un agente (docente, directivo, personal 
de apoyo o investigador), o grupo de agentes, que se ocupa de cuestiones locales, de bajo costo, factores fundamentales en el éxito de una innovación determinada. La segunda parte, en la que se integran criterios que incorporan un conocimiento especializado surgido de la investigación, en la que se hace uso del conocimiento acumulado sobre las innovaciones, y deberá servir de orientación a un equipo de especialistas que acompañará e investigará los procesos de innovación para generar un conocimiento propio de la institución sobre sus innovaciones. En especial, porque para avanzar en la formación de una cultura de la innovación se requiere de una comunidad que no sólo encuentra nuevas soluciones a los problemas vivos de la institución, sino que anticipa respuestas a nuevos desafíos. Y eso sólo se puede lograr si se cuenta con un conocimiento de la institución que no por local es menos riguroso. Por supuesto, el modelo tendrá que evolucionar conforme las condiciones del IPN lo demanden.

La primera parte del modelo se construyó tomando en cuenta algunos de los modelos que más aplicación han tenido, (Havelock y Huberman, 1977; Marín y Rivas, 1984; Tejada, 1998; De la Torre, 1997). Se toman en cuenta también las tres etapas del modelo de resolución de problemas (Problematización, Instrumentación y Evaluación) pero, como afirma De la Torre (1997), estas etapas no son independientes y no ocurren de manera lineal. Sin embargo, hay un énfasis en la problematización que aparece una y otra vez a lo largo del proceso, apelando a la creatividad de la red responsable de la innovación, para identificar y resolver los muchos problemas que surgen naturalmente en un proceso complejo. Por la importancia que reviste para el proceso, se comienza con la decisión de innovar, que comprende tres puntos:

- Los objetivos que se persiguen.

- Los doce criterios para caracterizar una innovación educativa.

- La relación efectiva de estos criterios con el cambio real mediante la definición de indicadores auténticos.

Y comprende ocho fases, que no necesariamente se siguen en el orden anotado, que conforman un ciclo que se reinicia para continuar en espiral en ciclos subsecuentes de mejora permanente. En cada una de las fases se pueden formar bucles, lo mismo puede ocurrir con los conjuntos de fases. 


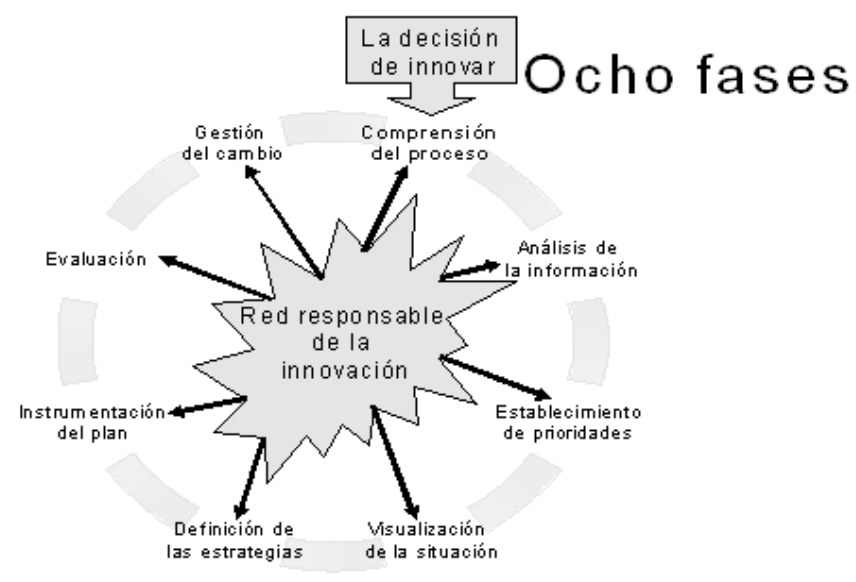

Figura 2. La decisión de innovar y las ocho fases del MIE del IPN.

En cada fase es recomendable elaborar una lista de verificación que comprenda dos aspectos:

- Preguntas básicas.

- Acciones fundamentales.

Además, en cada fase se sintetizan los datos generados, de preferencia en algún espacio virtual que permita a todos los involucrados aprovecharlos para aportar sugerencias durante el proceso de innovación, y se definen las necesidades de formación que suelen surgir constantemente y deben ser atendidas de manera oportuna.

En la que se puede llamar fase o, se trata básicamente de la decisión de innovar. Dado que la innovación necesitará el esfuerzo concertado y prolongado de varios agentes, entre otras cosas, la decisión de emprender la innovación es un aspecto que merece una consideración especial. Esta decisión de innovar requiere, de los responsables de la innovación, que sean capaces de visualizar con claridad lo que ocurrirá durante y, sobre todo, después del proceso de cambio que propiciará la innovación. Así, definiendo los objetivos y viendo la innovación a través de los criterios, se identifican aquellos aspectos que se pueden convertir en indicadores auténticos para verificar que la innovación realmente haya producido el cambio que se buscaba y haya acarreado las mejoras que se habían anticipado como consecuencia de la innovación. 
La importancia que ha adquirido la innovación educativa como parte de las políticas que se proponen mejorar la calidad y la pertinencia de la educación hace que no se pueda trivializar como algo que depende sólo de las buenas intenciones de un profesor insatisfecho o de un directivo emprendedor, o que puede ser impuesto desde la autoridad. Por ello, la decisión de emprender la innovación se convierte, en sí misma, en un proceso que permite precisar las características de la innovación $\mathrm{y}$, de ser necesario, modificarla antes de comenzar propiamente el proceso de innovación.

Como una ilustración del tipo de preguntas básicas y acciones fundamentales que comprende la lista de verificación de cada fase, se incluyen algunas preguntas y acciones de la primera fase, pero será la red responsable de cada innovación la que formule las preguntas y defina las acciones pertinentes a su innovación.

\section{LAS FASES DEL MODELO DE INNOVACIÓN EDUCATIVA}

\section{La comprensión del proceso de innovación}

Una vez tomada la decisión de innovar, la primera fase se concentra en la comprensión de la innovación, es decir, del proceso que la llevará a convertirse en la nueva normalidad. Puesto que se ha visto la innovación desde los doce criterios en la decisión de innovar, ya se cuenta con una definición de la innovación, así que, en esta primera fase, se requiere de información, datos, sobre la situación actual. Además, de la información sobre el contexto, se requiere también de información sobre las necesidades de formación, particularmente en el equipo responsable, que se han detectado, para hacer un plan y cubrirlas oportunamente.

\section{Las preguntas básicas}

- ¿Comparten todos los integrantes de la red una visión global del resultado de la innovación? ¿Tienen claro cuál es el papel que desempeñarán y qué responsabilidades tendrán que asumir?

- ¿Tiene la red de innovadores capacidad para propiciar el cambio? ¿En cuánto tiempo?

- ¿De qué información se dispone? ¿Cuál es la información que se necesita para monitorear el proceso y evaluar el cambio?

- ¿Cuál es la actitud con respecto a la innovación de todos los que participan en, o se ven afectados por, el proceso de la innovación?

- ¿Hay en la red responsable de la innovación equipos capacitados para la gestión del conocimiento y el análisis de datos? 


\section{Las acciones fundamentales}

- Identificar a todos los que participan en la innovación, o se ven afectados por ella, ya sea como promotores, participantes, asesores o beneficiarios.

- Definir con claridad el papel que le toca desempeñar a cada uno de ellos y describir el cambio que se espera que ocurra desde la perspectiva de cada uno.

- Conformar una red responsable de la innovación, procurando que haya representantes de todas las figuras que intervengan, muy probablemente todas las que hay en el Instituto.

- Establecer las necesidades de formación que se desprenden del punto anterior y preparar un cronograma para cumplirlas oportunamente.

- Hacer una descripción de los aspectos que se van a modificar utilizando los mismos indicadores que servirán para evaluar y monitorear la situación, y evaluar el resultado de la innovación.

- Establecer las necesidades de información sobre todos los aspectos pertinentes, identificar las fuentes donde conseguirla y estipular los plazos para hacerlo.

\section{El análisis de la información}

Una vez que se ha logrado una primera comprensión de lo que representa la innovación, es necesario dedicar mucha atención a los datos disponibles sobre todos los aspectos que concurren en la innovación. Del análisis de esta información, que puede requerir la participación de especialistas, surgirán las fortalezas y debilidades de la situación. Sólo que ahora serán vistas a través de los datos y del análisis que se realiza sobre ellos. En esta fase se tiene la oportunidad de profundizar en el conocimiento de la situación gracias a las relaciones que revele el análisis de la información.

\section{El establecimiento de las prioridades}

A partir del análisis de los datos se obtiene una nueva visión de la situación, una redefinición de los problemas que conlleva la innovación. Esta redefinición da lugar a una configuración en la que hay que identificar los aspectos prioritarios, ponderados según las posibilidades de solución que revelen los datos. La complejidad de los problemas puede requerir la definición de varios liderazgos que se responsabilicen de cada problema y de articular los planes para llegar a una solución adecuada y oportuna, tratando de aprovechar la sinergia de la red. 


\section{La visualización de la situación}

Una descripción basada en datos, como la que se ha logrado, de la situación original brinda la oportunidad de visualizar una solución también caracterizada por datos. La profundización conseguida en las fases anteriores arroja una descripción más precisa de la situación que resultará del proceso de innovación. La definición de los indicadores y de los instrumentos que servirán para medirlos, junto con el establecimiento de prioridades, permiten establecer metas parciales, con plazos, que conducirán a lograr la situación que ahora se puede representar mediante matrices de datos.

\section{La definición de las estrategias}

Una vez que se ha visualizado la situación, mediante indicadores que darán lugar a datos, hay que establecer cómo se conseguirá que la innovación se realice. No hay que perder de vista que, si los indicadores definidos son auténticos, es la evolución de los datos la que debe servir de referencia durante el proceso, mediante los monitoreos, y que las estrategias tendrán que considerar esta evolución. Los aspectos que se consideran, desde una perspectiva integral y sistémica, valiosos, como las cuestiones éticas y emocionales, tendrán que tomarse en cuenta, de manera equilibrada, en las estrategias y en los indicadores si no se quiere correr el riesgo de caer en una visión sesgada que desvirtúe la innovación.

\section{La instrumentación del plan}

En la instrumentación del plan que conducirá a la realización de la innovación suelen surgir problemas nuevos que obligarán a la red responsable a revisar parte de los planes y a regresar a algunas de las fases anteriores. Esto es lo normal debido a la complejidad de los procesos de innovación. La flexibilidad de la organización en redes es un factor que se puede aprovechar, durante la instrumentación de los planes, para atender los imprevistos que nunca faltan y que pueden llegar a constituirse en obstáculos insuperables si no se anticipa la forma de enfrentarlos cuando surjan. Los datos y los instrumentos diseñados para generarlos desempeñan un papel fundamental si se quieren lograr resultados comprobables.

\section{La evaluación}

Una innovación sólo merece tal nombre cuando produce cambios en todos los niveles de la organización, desde los más visibles hasta los supuestos básicos, los 
que están en la base de las acciones y decisiones cotidianas. La evaluación acompaña al proceso de innovación de manera permanente. Pero sólo se pueden registrar como datos aquellos aspectos para los que existe un instrumento. No se puede mejorar lo que no se mide. Por esto es fundamental el monitoreo de los indicadores, para percatarse de las tendencias y ver si evolucionan en el sentido previsto, no necesariamente de manera lineal con el tiempo. La variedad de los indicadores (de resultados, de procesos, de estrategia) permite dar un seguimiento más matizado del proceso. En tanto se tiene conocimiento sobre el desarrollo de las innovaciones se pueden identificar patrones de evolución caracterizables (lineales o, como suele ser en estos casos, no lineales) que permiten formular hipótesis más precisas y dar seguimiento a los aspectos medibles. Se pueden incluir otro tipo de registros, como diarios y bitácoras, que resultan útiles cuando se hace la narrativa de la experiencia.

\section{La gestión del cambio}

Una innovación ha tenido éxito cuando se convierte en la nueva normalidad de la institución, cuando las personas han interiorizado un patrón nuevo. No hay, sin embargo, innovaciones irreversibles, por lo que hay que administrar el cambio cuando ha producido una mejora verificable. Cuban (1998) ha destacado la dificultad que hay para juzgar el éxito o el fracaso de una innovación porque las escuelas cambian las reformas tanto como las reformas cambian las escuelas.

La segunda parte del Modelo de Innovación Educativa del IPN complementa la primera cuando se ha decidido investigar el proceso de innovación y comprende una serie de criterios que aportan un conocimiento de base para realizar investigación sobre las innovaciones educativas.

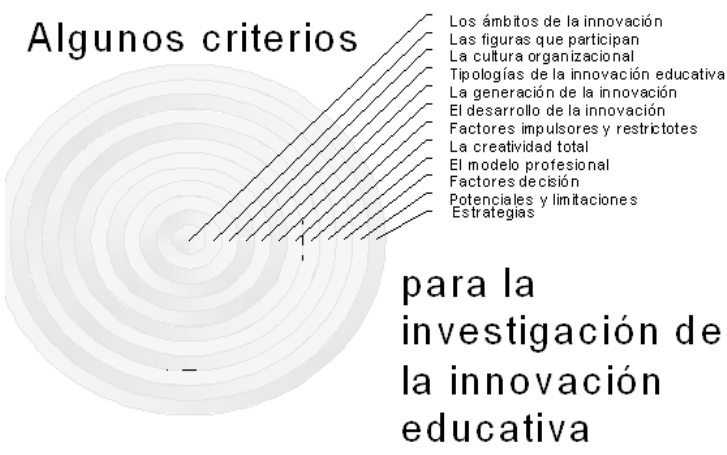

Figura 3. Algunos criterios para la investigación de la innovación educativa del Modelo de Innovación Educativa del CFIE 
En cada proyecto se identificará la línea de investigación pertinente y se utilizarán sólo los criterios que sirvan como marco del estudio, aportando el conocimiento que se haya acumulado en la comunidad de investigación sobre innovaciones educativas.

\section{LAS FIGURAS DE LA INNOVACIÓN}

El proceso de innovación puede ser considerado simultáneamente desde varios puntos de vista. Entre ellos se encuentra la innovación como un conjunto de personas e instituciones relacionadas por el papel que desempeñan en la innovación: promotores, participantes, asesores, investigadores, observadores.

La red responsable de la innovación es una organización en la que participan personas diferentes con papeles complementarios pero no excluyentes, que conforman un sujeto colectivo. En la red se pueden identificar a los promotores de la innovación, quienes tienen un interés en que la situación deseada se convierta en la nueva normalidad, en términos subjetivos se trata de las personas que están convencidas de las bondades de los cambios que acarreará la innovación. Los promotores cuentan con un conocimiento acerca de la innovación específica y están dispuestos a participar en los procesos de formación y actualización que se presenten en el proceso de innovación. Los promotores también están dispuestos a contribuir a la formación y el fortalecimiento de una cultura de la innovación en su medio.

Los asesores son elementos fundamentales en la red, pues aportan una gama de saberes multidimensionales sobre los diversos aspectos que concurren en la innovación educativa. Los asesores expertos en los procesos de innovación educativa son particularmente importantes pero también se requieren asesores en todos los aspectos que necesiten de conocimiento especializado, como pueden ser algunas cuestiones tecnológicas, disciplinarias, organizacionales, etcétera.

El observador externo desempeña un papel de registro y análisis del proceso. La observación es un proceso sistemático que se centra en los aspectos sustantivos de la innovación. Es preciso definir los objetos de la observación y las condiciones en que ocurrirá. Como proceso sistemático, la observación contará con una guía e instrumentos para su realización.

El investigador realiza un trabajo formal según sus intereses de estudio para generar un conocimiento específico sobre algún aspecto particular de la innovación. Aunque el grado de compromiso de quienes intervienen en una innovación es diverso, los participantes son personas que, como parte del proceso de innovación, se ven 
llevados a adoptar una posición fundamentada con respecto a la innovación. Y hay también quienes se ven favorecidos por el proceso o los resultados de la innovación en su relación con la institución educativa. Estos beneficiarios de la innovación no necesariamente forman parte de la red responsable.

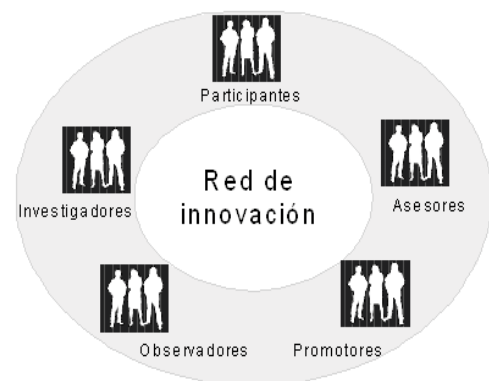

Figura 4. La red responsable de la innovación.

La organización de la red responsable depende del tipo de innovación. Sin embargo, se pueden dar algunas características generales de la estructura de la red. La red es horizontal, sin jerarquías fijas, con liderazgos flexibles en función de los retos particulares que surgen en el proceso de innovación. El talento humano se manifiesta mejor en un contexto complejo cuando la red se puede reorganizar continuamente. Los cambios implican una crítica, por lo que en las innovaciones siempre habrá personas que se sientan afectadas y, naturalmente, se generan conflictos. La administración de estos conflictos de manera responsable y flexible es una de las tareas que la red realiza permanentemente a lo largo del proceso de innovación.

Como parte de los esfuerzos de vinculación con diversas instancias de la sociedad, es importante incluir a representantes de sectores como el industrial, gubernamental y de organizaciones sociales. Pueden aportar, desde sus perspectivas, una mirada que haga visibles aspectos de la innovación que, de otra manera, podrían pasar inadvertidos.

\section{LA CULTURA DE LA INNOVACIÓN}

La formación y el desarrollo de una cultura de la innovación requieren de una reflexión sistemática sobre el efecto acumulado de múltiples innovaciones 
educativas, por un lado, y de estrategias para la modificación de prácticas, pautas, valores y supuestos personales y organizacionales, por otro lado. En las instituciones que carecen de una tradición en la innovación educativa es preciso lograr que toda la comunidad se integre en diversas redes responsables de proyectos de innovación para que viva la experiencia de reflexión, acción y evaluación de su quehacer que implican las innovaciones. Pero, además, una institución que quiere crear una cultura de la innovación, como afirma Tristá, se espera que "institucionalice la innovación; dedique espacio, tiempo y dinero para el desarrollo organizacional; establezca unidades de generación y experimentación con una función de investigación y desarrollo; provea recompensas para los innovadores; instale mecanismos de rastreo del ambiente para conocer nuevos desarrollos en temas de su interés" (2004, pp. 426-427). Dado que la cultura constituye un sistema que integra, de manera compleja, creencias, conocimientos, actitudes, hábitos mentales y costumbres, en el que las personas viven en sociedad, los cambios en este nivel requieren de estrategias igualmente complejas que, en periodos más bien prolongados, modifiquen el nivel de supuestos y premisas básicas que se encuentra en la base de los patrones de conducta.

En muchos casos, las propuestas innovadoras son una respuesta de los centros a una determinada política educativa dela institución a la que pertenecen. Esta situación lleva a que las innovaciones no se anticipen ni abran caminos a la política educativa. Esta tendencia, natural en instituciones con poca tradición en la innovación, puede cambiar cuando se propone el desarrollo de una cultura de la innovación que propicie la generación de innovaciones en distintas instancias del sistema.

En una reunión de especialistas, organizada por la UNESCO sobre La cultura de la innovación y la construcción de la sociedad del conocimiento (Bovermann y Russell, 2004) se establecieron dieciocho puntos que servirán de referencia para los estudios que se realicen sobre este tema. En esta declaración se afirma que una cultura de la innovación, vinculada de manera estrecha con la noción de sociedad del conocimiento, comprende por lo menos una dinámica social, expresiones de autorreflexión, tolerancia, respeto a la diversidad, la consideración explícita de la gestión del conocimiento y un conjunto de aspiraciones colectivas que apuntan al desarrollo de la creatividad. No se puede ignorar que la cultura de la innovación no es un fin en sí misma y que cada institución tiene que identificar los valores éticos y sociales que sostienen su propia cultura de la innovación.

Sancho et al. (1998), a partir de tres estudios sobre innovaciones, identifican algunas características de una cultura de la innovación. A continuación se describe la cultura de la innovación aprovechando ambos trabajos. 
La globalización es una consecuencia de innovaciones en distintos campos. De aquí la importancia del fomento de una cultura de la innovación. De esta forma, cuando se tiene un cuestionamiento de las prácticas actuales y los logros obtenidos, se reflexiona y discute sobre ellos para buscar alternativas de mejora. Alternativas que toman la forma de innovaciones.

Una cultura de la innovación alienta a las personas a trabajar en innovaciones, sin esperar que todas éstas provengan de la institución en la que se labora. Y es que todos están acostumbrados a sugerir mejoras y modificaciones continuas y replanteamientos institucionales. Se crea un ambiente al interior de las instituciones en el que todas las personas se sienten parte activa de las innovaciones. Además, durante el desarrollo de las innovaciones se da una reelaboración colectiva de las informaciones individuales con la consiguiente modificación de indicadores y supuestos. Es común que en los equipos surjan diferencias y se requieran reorganizaciones. Pero las innovaciones crean nuevas necesidades y abren nuevas perspectivas profesionales.

En la sociedad del conocimiento las necesidades de aprendizaje son permanentes y se reconoce la importancia del aprendizaje autónomo. Se vive en un proceso de formación continua a consecuencia de los desequilibrios constantes que acarrean los cambios en las instituciones educativas. Entre los individuos se presentan reacciones variadas. Algunos incorporan la necesidad permanente de aprendizaje en sus esquemas de funcionamiento, pero otros se muestran reacios, pues necesitan aprender a enfrentar la incertidumbre que se genera. Las innovaciones en una institución educativa constituyen un sistema óptimo de formación permanente.

Así pues, no sólo es importante impulsar el desarrollo de innovaciones, también lo es la creación de una cultura de la innovación que permita arraigar en la comunidad esa dinámica que impulse a la institución a una mejora permanente, a no esperar que los cambios la alcancen y avasallen, sino a estar preparados y ser de los primeros en impulsar y aprovechar tales cambios.

\section{APLICACIÓN DEL MODELO DE INNOVACIÓN EDUCATIVA}

Una incubadora de innovaciones educativas es un ambiente en el que una red de innovadores tiene acceso a las herramientas, recursos, conocimiento y asesoría que requiere para realizar una innovación hasta institucionalizarla e interiorizarla, convirtiéndola en la nueva normalidad. Se busca tener condiciones óptimas, mediante el control de algunas variables que permitan intensificar partes del proceso para obtener resultados en un tiempo más breve. 


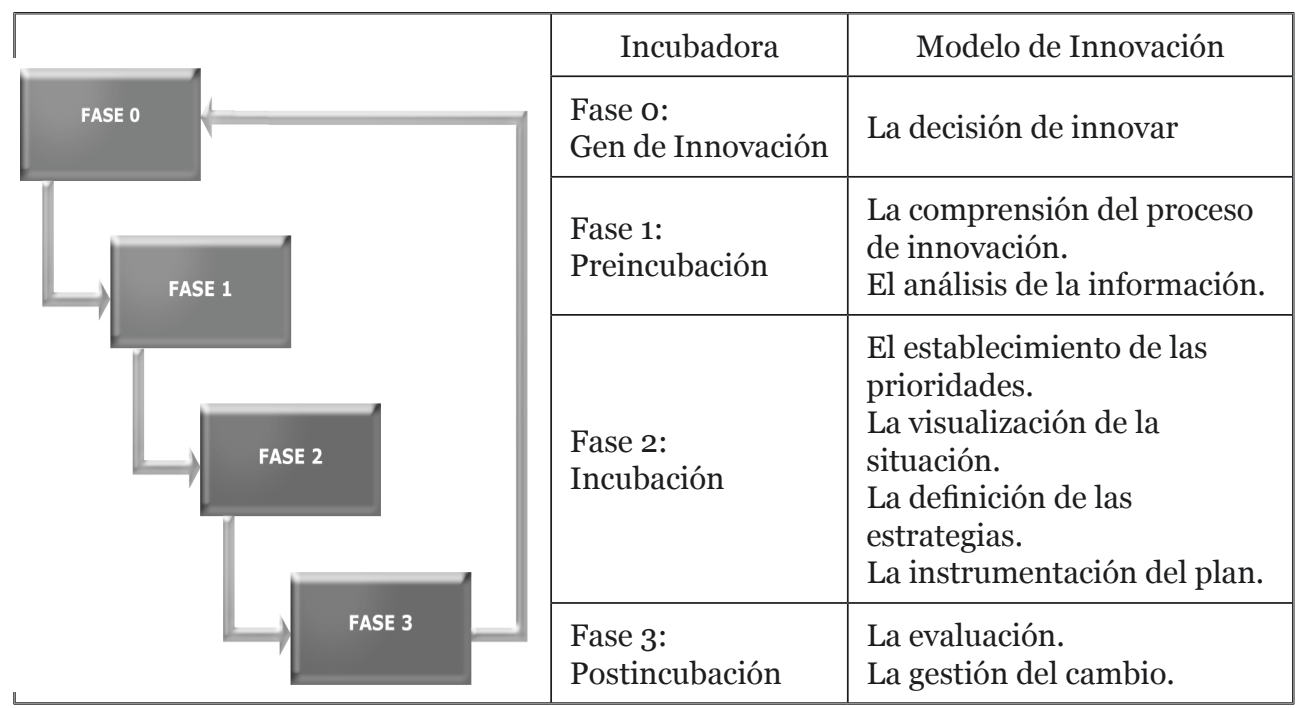

Tabla 2. Correspondencia entre las fases de incubación y las del MIE.

Como se muestra en la tabla anterior hay una correspondencia entre las fases del proceso de incubación y las fases del Modelo de Innovación Educativa.

En una reforma académica tan ambiciosa como la que emprendió el IPN se reconoce a la profesionalización docente como un elemento fundamental para consolidar una educación de alta calidad. El seminario Repensar las Matemáticas constituye una innovación que sienta las bases para una solución de fondo al problema de la falta de calidad y pertinencia de la educación. El seminario RM contribuye a la profesionalización docente de los profesores de matemáticas, particularmente en la vinculación de los resultados de la investigación en matemática educativa con la práctica docente y en el fortalecimiento de la colegialidad a través de la integración de una comunidad virtual que se ocupa de problemas vivos en la matemática educativa y en la práctica docente, desde las perspectivas del investigador y del profesor. Como proyecto de innovación educativa, en el seminario RM las fases y los criterios del Modelo de Innovación Educativa del IPN han permitido identificar los logros, para consolidarlos y rediseñar algunas partes, como la estructura de las sesiones, que se enfoquen al logro de los cambios más difíciles. 


\section{REFERENCIAS BIBLIOGRÁFICAS}

Blanco, R.; Messina, G. (2000). Estado del arte sobre las innovaciones educativas en América Latina. Colombia: Convenio Andrés Bello-UNESCO.

Bovermann, T.; Russell F. W. (2004). A Culture of Innovation and the Building of Knowledge Societies. París: UNESCO.

CFIE. (2006). ¿Quiénes somos? [en línea]. Disponible en: http://www.cfie.ipn. $\mathrm{mx} /$ Nosotros/CFIE.html [consulta 2006, 18 de octubre]

Cuban, L. (1998). How schools change reforms: Redefining reform success and failure. Teachers College Record, 99, 453-477. [en línea] Disponible en: http://www.tcrecord.org/Content. asp?ContentId=10273 [consulta 2006, 18 de octubre]

De la Torre, S. (1997). La innovación educativa. España: Editorial Dykinson.

Fullan, M.; Stiegelbauer, S. (1997). El cambio educativo. México: Editorial Trillas.

Havelock, R. G.; Huberman, A. M. (1977). Solving educational problems: the theory and reality of innovation in developing countries. Suiza: UNESCO.

IPN. (2004). Materiales para la reforma. Publicaciones 01 a 19 [en línea]. Disponible en: http://www.mreforma. ipn.mx/ [consulta 2006, 18 de octubre]

IPN. (2004a). Materiales para la reforma. Publicación o1 Un Nuevo Modelo Educativo para el IPN. [en línea]. Disponible en: http://www.mreforma. ipn.mx/ [consulta 2006, 18 de octubre]

IPN. (2004b). Materiales para la reforma. Publicación o6 Modelo de Integración Social del IPN Programa Estratégico de Vinculación, Internacionalización y Cooperación. [en línea]. Disponible en: http://www.mreforma.ipn.mx/ [consulta 2006, 18 de octubre]

IPN. (2004c). Materiales para la reforma.
Publicación 02 Diagnóstico por Comparación (Benchmarking) Aplicado a Instituciones del Nivel Medio Superior de México. [en línea]. Disponible en: http://www.mreforma. ipn.mx/ [consulta 2006, 18 de octubre]

IPN. (2004d). Materiales para la reforma. Publicación o3 Diagnóstico por Comparación (Benchmarking) Aplicado a Instituciones de Educación Superior de México. [en línea]. Disponible en: http://www.mreforma. ipn.mx/ [consulta 2006, 18 de octubre]

IPN. (2004e). Materiales para la reforma. Publicación 05 Diagnóstico por Comparación (Benchmarking) Aplicado a Instituciones de Investigación y Nivel de Posgrado de México. [en línea]. Disponible en: http://www.mreforma.ipn.mx/ [consulta 2006, 18 de octubre]

Ramírez, M. E.; Torres, J. L.; Suárez, L.; Ortega, P. (2006). Vínculos entre la investigación y la práctica en la matemática escolar del IPN: El Seminario Repensar las Matemáticas, una innovación en la formación docente. Memorias de Virtual Educa 2006. Bilbao, España 2006 [en línea]. Disponible en: http://somi. cinstrum.unam.mx/virtualeduca2006/ pdf/110-MRS.pdf [consulta 2006, 7 de septiembre]

Sancho, J. M.; Hernández, F.; Carbonell, J.; Tort, T.; Simón, N.; SánchezCortes, E. (1998). Aprendiendo de las innovaciones en los centros. España: Octaedro.

SEP. (2001). Glosario. Subsecretaría de Planeación y Coordinación [en línea]. Disponible en: http://www.sep.gob. mx/work/appsite/pubbasoo/index. htm [consulta 2006, 17 de octubre]

Suárez, L.; Cordero, F.; Daowz, P.; Ramírez, A.; Ortega, P.; Torres, J. L.; Romano, 
S.; Servín, C.; Téllez, J.; Domínguez, B. (2006). De los paquetes didácticos hacia un repositorio de objetos de aprendizaje: un reto educativo en matemáticas. Memorias de Virtual Educa 2006. Bilbao, España [en línea] Disponible en: http://somi. cinstrum.unam.mx/virtualeduca2006/ pdf/124-LST.pdf [consulta 2006, 7 de septiembre]

Tejada, J. (1998). Los agentes de innovación en los centros educativos. Málaga: Aljibe.

Tristá, B. (2004) Introducción a la administración académica. México: UAM-X.

UNESCO (2001). Red de Innovaciones Educativas para América Latina y el Caribe. [en línea]. Disponible en: http://innovemos.unesco.cl/ [consulta 2006, 18 de octubre]

\section{PERFIL ACADÉMICO Y PROFESIONAL DE LOS AUTORES}

Pedro Ortega Cuenca. miembro fundador de la Academia Institucional de Matemáticas (AIM-NMS-IPN) y asesor desde su fundación en 1995 hasta 2005. Coordinador del proyecto Paquetes Didácticos de Matemáticas, proyecto cuyo propósito fue el diseño de materiales educativos para los seis cursos de Matemáticas de acuerdo a los planes de estudio del IPN de 1994 en formato impreso, disco compacto y sitios en Internet y plataformas educativas.

E-mail: poc52052@prodigy.net.mx

María Eugenia Ramírez Solís, Profesora del IPN con estudios de maestría en Comunicación y Tecnologías Educativas, ha diseñado, coordinado e impartido Programas de Formación Docente y de Diseño y Desarrollo Curricular a través del Departamento de Pedagogía. Asesor académico en el programa de mejoramiento de programas de estudio de la Universidad Autónoma de la Ciudad de México (UACM). Integrante de la red académica para el Seminario Repensar las Matemáticas.

E-mail:meramire@gmail.com

José Luis Torres Guerrero, profesor de bachillerato en el IPN. Tiene grado de Maestría en Ciencias en la especialidad de Matemática Educativa. Formó parte de la Academia Institucional de Matemáticas del Nivel Medio Superior del IPN (1994-2005) y participó en proyectos de la misma, por ejemplo en la elaboración de Paquetes Didácticos de Matemáticas y en el ciclo de videoconferencias Repensar las Matemáticas. Ha participado en congresos presentando ponencias y como tallerista.

E-mail: jeluistg@yahoo.com.mx 
Citlali Yacapantli Servín Martínez, Ingeniera en Biónica del Instituto Politécnico Nacional (IPN), actualmente es alumna de la Maestría en Ciencias en Matemática Educativa del Centro de Investigación y de Estudios Avanzados del IPN. Ha impartido clases en el nivel medio superior y en talleres de formación docente. Tiene experiencia en proyectos relacionados con redes de colaboración y educación a distancia. Interés en el uso de las TIC's para la enseñanza-aprendizaje de las Matemáticas.

\section{E-mail: cservin@cinvestav.mx}

Liliana Suárez Téllez, actualmente desarrolla una investigación doctoral sobre el uso de las gráficas en la modelación del cambio en el Departamento de Matemática Educativa del Centro de Investigación y de Estudios Avanzados del IPN. Ha diseñado y participado en Programas de Formación Docente en matemáticas en el IPN y en algunos estados de la República Mexicana y el extranjero. Fue responsable del Portal de la Academia Institucional de Matemáticas: http://www.comunidades.ipn.mx/ aim desde su creación hasta 2005.

E-mail: 1suarez@ipn.mx

Blanca Ruiz Hernández actualmente realiza estudios de doctorado sobre la variable aleatoria en la Facultad de Educación de la Universidad de Granada bajo la dirección de Carmen Batanero. Es profesora del Institututo Tecnológico de Monterrey, Campus Monterrey. Es Chair del Comité Organizador de la Joint ICMI IASE Study que se realizará en Monterrey en 2008.

E-mail: bruiz@itesm.mx

Ana Emilia López Rayón Parra, profesora de tiempo completo del Instituto Politécnico Nacional, obtuvo la licenciatura y especialidad en psicología de la Universidad Autónoma de México, ha participado como diseñadora e instructora en diversos diplomados, cursos, talleres y seminarios sobre educación y nuevas tecnologías ha realizado lagunas investigaciones educativas y cuenta con experiencia en desarrollo curricular y de formación de profesores. Actualmente impulsa proyectos de innovación educativa y de desarrollo de aprendizaje virtual.

E-mail: aelopez@ipn.mx

DIRECCION DE LOS AUTORES

Pedro Ortega Cuenca

Jacarandas 94-6, Santa María Insurgentes,

Del. Cuauhtémoc, México D.F. 06430

México 


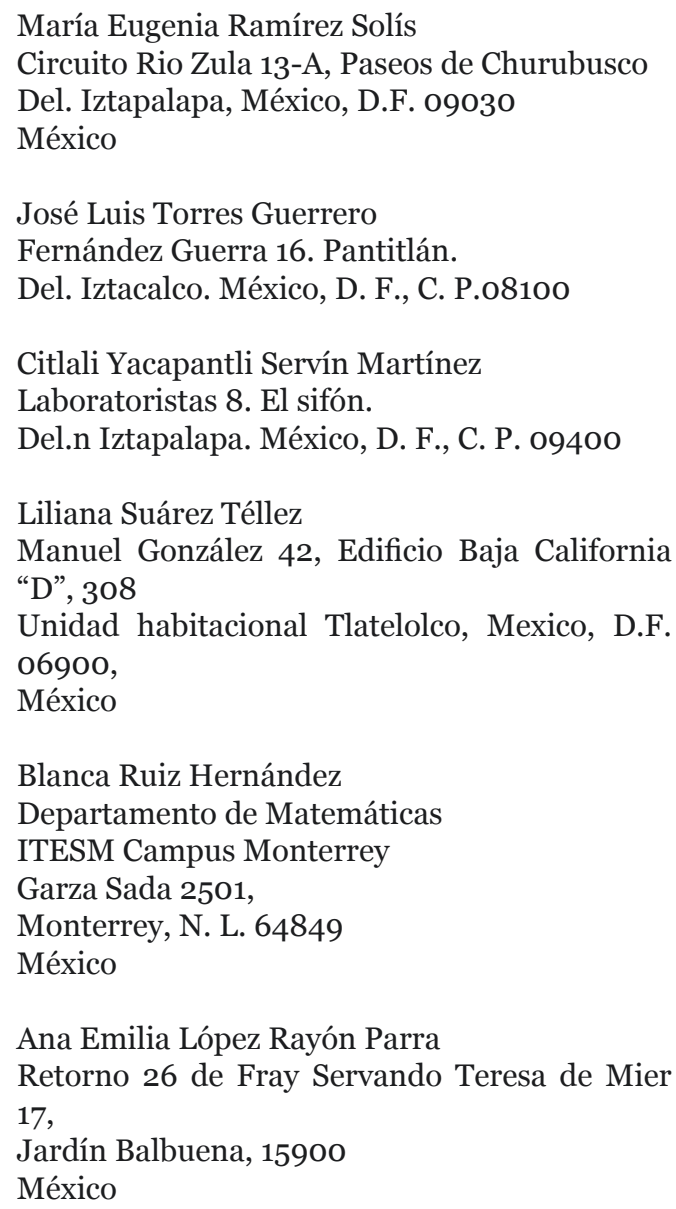

Fecha de recepción del artículo: 23/10/06 Fecha de aceptación del artículo: 15/03/07 